Meta

Journal des traducteurs

Translators' Journal

\title{
Réglons donc ce cas !
}

\section{Paul C. Abinakle}

Volume 32, numéro 4, décembre 1987

URI : https://id.erudit.org/iderudit/001849ar

DOI : https://doi.org/10.7202/001849ar

Aller au sommaire du numéro

Éditeur(s)

Les Presses de l'Université de Montréal

ISSN

0026-0452 (imprimé)

1492-1421 (numérique)

Découvrir la revue

Citer cet article

Abinakle, P. C. (1987). Réglons donc ce cas ! Meta, 32(4), 448-450.

https://doi.org/10.7202/001849ar

Ce document est protégé par la loi sur le droit d'auteur. L’utilisation des services d'Érudit (y compris la reproduction) est assujettie à sa politique d'utilisation que vous pouvez consulter en ligne.

https://apropos.erudit.org/fr/usagers/politique-dutilisation/
Cet article est diffusé et préservé par Érudit.

Érudit est un consortium interuniversitaire sans but lucratif composé de l’Université de Montréal, l'Université Laval et l'Université du Québec à Montréal. Il a pour mission la promotion et la valorisation de la recherche. https://www.erudit.org/fr/ 


\section{RÉGLONS DONC CE CAS!}

On rencontre souvent dans les textes d'assurance le mot anglais case, comme dans " to settle a case" ou " to submit a case", etc. Les dictionnaires et vocabulaires d'assurances (unilingues ou bilingues) sont muets à ce sujet. Comment devons-nous donc traduire case en français?

Pour certains, le problème ne se pose même pas. Ils traduisent ce mot par (quoi d'autre ?) cas . On voit ainsi des cas soumis, des cas réglés... Non seulement ces traductions sont fausses et calquées, mais elles prêtent inutilement à confusion. Voici pourquoi.

A part le sens grammatical que nous écartons, le mot français cas peut signifier :

1. Ce qui arrive ou peut arriver.

2. Situation définie par la loi pénale. 
3. Difficulté sur un point de morale, de religion.

4. État et évolution de l'état d'un sujet, du point de vue médical ${ }^{1}$.

Or il n'est question ici ni d'éventualité, ni de morale, ni de religion, ni de maladie, ni de situation définie par la loi pénale. D'autres persistent à donner à cas un sens juridique pour justifier son emploi dans des textes qui portent sur les assurances. Examinons la valeur de ce raisonnement.

Si nous donnons à cas un sens juridique en français, il faut bien lui donner le même sens en anglais aussi. Case signifiera alors " $A$ suit or action in law or equity: the evidence supporting a conclusion or judgment $»^{2}$.

Voici deux exemples courants où case est souvent traduit pas cas : 1) "The agent must settle 15 cases a month in order to qualify... ";2) "Below are the underwriting statistics for the month of August, showing received business. Also included are to date statistics for rated, postponed and not take cases. " En traduisant case par cas dans ces deux phrases, on se demande s'il est question d'un agent d'assurances ou d'un avocat à la Cour!

Nombre de traducteurs font cette confusion entre case et son homographe français cas, soit parce qu'ils donnent à ce dernier mot une extension qu'il n'a pas en réalité, soit parce qu'ils lui donnent un sens prétendument juridique. Pourquoi "prétendument "? Parce qu'il se trouve que, même pris dans un sens juridique, cas est un mot «dont la forme est suspecte et doit être remplacé par litige, poursuite, cause, etc. $»^{3}$. Mais on empiète là sur un autre sujet...

Maintenant que nous avons écarté l'emploi de cas, par quoi allons-nous traduire case? Voyons tout d'abord le sens de case dans le domaine qui nous intéresse. Case n'a pas de sens précis en assurances (bien qu'il soit largement utilisé dans ce domaine). Le sens le plus plausible serait : "The object of investigation or consideration $)^{2}$, sens que le mot cas n'a pas en français. Qu'est-ce qui fait l'objet d'une étude, d'une enquête en assurance ? On est assez vite porté à déduire qu'il s'agit de la proposition d'assurance, ce qui est vrai (du moins en partie). Cependant, il faut savoir que case est un de ces mots passepartout qui peut avoir mille et un sens. "There is perhaps no single word so freely resorted to as a trouble-saver, and consequently responsible for so much flabby writing. " ${ }^{4}$

S'agit-il donc de la proposition ou du contrat d'assurance ? L'anglais ne fait pas cette distinction nette entre la proposition soumise à la compagnie d'assurance et le contrat qui est délivré au client après l'approbation de l'assureur. Case englobe les deux notions.

À mot passe-partout en anglais, un mot passe-partout en français. Le mot affaire peut nous être d'un grand secours. On sait qu'affaire traduit business et qu'il a le sens des « assurances souscrites ou en cours de souscription, envisagées à un stage particulier du processus de production ou d'administration ${ }^{5}$. Case serait donc un synonyme (peut-être imparfait, mais un synonyme quand même) de business. Les deux mots ont une extension sémantique très large et leurs champs de signification se recoupent souvent. Bien des fois l'anglais utilise indifféremment cases et business. Parfois, policies et contracts viennent s'ajouter à la liste. Notons que le mot affaire est le plus souvent utilisé au pluriel. De plus, c'est un pluriel de volume, de quantité, de production, par opposition à un pluriel numérique. "La compagnie a réalisé d'excellentes affaires " se dit et se lit très bien. Mais l'œil est en quelque sorte choqué par une phrase comme " la compagnie a réalisé (ou réglé) quarante affaires ». C'est le mot contrat (ou police) qui vient spontanément à l'esprit. On dit couramment qu'une affaire est réglée, classée, etc. Il reste que ce mot est le plus souvent utilisé au pluriel dans la terminologie des assurances. Il est intéressant de signaler que même si l'anglais emploie business et cases dans le même sens, il réserve business pour désigner un montant, un volume et utilise cases 
lorsqu'il s'agit d'un nombre. Exemple : "From May 1 to Aug. 15 the business received was $3500000 \$$ (or 109\% of objective). The business settled was $2500000 \$$ (or $75 \%$ of objective). The cases received were 11578 and the settled cases $8557 \ldots$ in the event of a tie, the agent settling the highest number of cases (et non business) will be declared... ".

On peut donc traduire case par affaire. Les "submitted cases, settled cases" sont les affaires soumises, réglées. Un autre équivalent utile est dossier. Les "rated cases" peut être rendue par dossier des risques aggravés. Parfois, il est inutile de rendre case. "Each year there have been some problems with RRSP cases around the end of the year... ": Chaque année, les REER police. "Agents must have had earnings of $35000 \$$ plus settlement of at least 25 separate cases" : les agents doivent avoir accumulé $35000 \$$ en commissions et avoir fait souscrire 25 contrats différents. Si le contexte est assez clair, on peut également traduire case par proposition. En somme, ce qu'il faut retenir, c'est que case est un mot passe-partout et que sa traduction doit être faite en situation, comme on dit en linguistique.

\section{RÉFÉRENCES}

Références

1. P. Robert (1986) : le Petit Robert, Dictionnaire le Robert, Paris.

2. Webster (1977) : Webster's New Collegiate Dictionary, Springfield, Massachusetts, Merriam Co.

3. W. Schwab (1984) : les Anglicismes dans le droit positif québécois, Québec, Éditeur officiel du Québec.

4. H.W. Fowler (1982) : A Dictionary of Modern English Usage, New York, Oxford University Press.

5. L.-P. Béguin (1979) : Vocabulaire technique des assurances sur la vie, Montréal, Office de la langue française.

Paul C. Abinakle Toronto, Canada 